\title{
Correspondence
}

\section{Renal impairment in relation to non-steroidal anti-inflammatory drugs}

SIR, Unsworth et al have recently shown that asymptomatic, reversible impairment of renal function is common in patients with rheumatoid arthritis (RA) receiving long term non-steroidal anti-inflammatory drug (NSAID) treatment. ${ }^{1}$ This conclusion was derived from the observation that when NSAID treatment in 11 patients was stopped there was a significant rise in creatinine clearance, a significant fall in serum creatinine, and a trend downwards in serum urea, compared with 11 patients who continued to receive NSAIDs, in whom no such changes were seen.

We have recently undertaken a study to investigate the effects of a new NSAID (tenoxicam) on renal function in a larger number of patients. The study comprised 40 patients (four male, 36 female; mean age (SEM) 72 (1.4) years) with RA or osteoarthritis and suffering from renal impairment as defined by a creatinine clearance of $25-80 \mathrm{ml} / \mathrm{min}$. Patients stopped their NSAID treatment for a three day $(n=27)$ or seven day $(n=13)$ washout period before starting tenoxicam ( $20 \mathrm{mg} / \mathrm{day})$, which was continued for 12 weeks. All other drug treatment was continued as before such that low dose prednisolone was continued by seven patients and second line agents by eight. Renal function was monitored by measurement of creatinine clearance, serum creatinine, serum urea, and urine $\beta-N$ acetylglucosaminidase/creatinine ratio at baseline (time of stopping current NSAID), week 0 (time of starting tenoxicam treatment) and weeks 4,8 , and 12 of tenoxicam treatment.

The Table shows a marked improvement in renal function during the washout period with a gradual retur towards the baseline status during the period of tenoxicam treatment. In the case of serum creatinine and serum urea the mean results at week 12 were essentially the same as a baseline, whereas creatinine clearance remained higher than baseline, though not significantly.

In Unsworth's study the time patients were not receiving NSAIDs varied from three to 36 days, whereas in our stud $\bar{\Phi}$ patients were only without NSAIDs for three or seveit days. The 27 patients who only had a three day washout still showed a significant rise in creatinine clearanck $(p<0.01)$. This makes the effect of NSAID withdrawal oP renal function even more pronounced and suggests क prostaglandin mediated mechanism. Our results in a larger patient series support the observations of Unsworth Furthermore, our results indicate that the effect of up to 1 s weeks of tenoxicam treatment on patients with rena impairment was mild and certainly no worse than for other NSAIDs as judged by comparison of results at week $1 \overrightarrow{20}$ with those at baseline, which presumably reflect the degre $\infty$ of renal impairment induced by the previous NSAIB treatment. The latter would clearly vary in choice, doseo and duration from patient to patient.

Clinical Pharmacology Unit,

(Rheumatism Research),

Royal Bath Hospital,

Cornwall Road,

Harrogate HG1 2PS

\section{Reference}

1 Unsworth J, Sturman S, Lunec J, Blake D R. Renal impais ment associated with non-steroidal anti-inflammatory drugs. Ann Rheum Dis 1987; 46: 233-6.

Table Changes in renal function parameters after stopping NSAIDs and starting tenoxicam treatment

\begin{tabular}{|c|c|c|c|c|c|c|c|c|c|c|c|c|c|}
\hline \multirow[t]{2}{*}{ Week No } & \multirow{2}{*}{$\begin{array}{l}\text { No of patients } \\
\text { continuing to } \\
\text { receive tenoxicam }\end{array}$} & \multicolumn{3}{|c|}{$\begin{array}{l}\text { Creatinine clearance } \\
(\mathrm{ml} / \mathrm{min})\end{array}$} & \multicolumn{3}{|c|}{$N A G^{\dot{r}} /$ creatinine } & \multicolumn{3}{|c|}{$\begin{array}{l}\text { Serum creatinine } \\
\text { ( } \mu \mathrm{mol} / \mathrm{l})\end{array}$} & \multicolumn{3}{|c|}{$\begin{array}{l}\text { Serum urea } \\
\text { (mmol/l) }\end{array}$} \\
\hline & & $n$ & $\bar{x}$ & $S E M$ & $n$ & $\bar{x}$ & SEM & $n$ & $\bar{x}$ & $S E M$ & $n$ & $\bar{x}$ & $S E M$ \\
\hline $\begin{array}{l}\text { Baseline } \\
0 \\
4 \\
8 \\
12\end{array}$ & $\begin{array}{l}-40 \\
39 \\
28 \\
19\end{array}$ & $\begin{array}{l}37 \\
33 \\
23 \\
19 \\
15\end{array}$ & $\begin{array}{l}48 \cdot 3 \\
62 \cdot 4 \\
67 \cdot 6 \\
66 \cdot 9 \\
58 \cdot 1\end{array}$ & $\begin{array}{l}2 \cdot 2 \\
3 \cdot 8 \\
4 \cdot 7 \\
4 \cdot 9 \\
7 \cdot 7\end{array}$ & $\begin{array}{l}(6) \\
40 \\
27 \\
22 \\
17\end{array}$ & $\begin{array}{c}(139) \\
91 \\
75 \\
74 \\
78\end{array}$ & $\begin{array}{c}(39) \\
10 \\
7 \\
10 \\
12\end{array}$ & $\begin{array}{l}39 \\
38 \\
30 \\
23 \\
19\end{array}$ & $\begin{array}{l}104 \\
89 \cdot 5 \\
92 \cdot 0 \\
102 \\
105\end{array}$ & $\begin{array}{l}4 \cdot 3 \\
4 \cdot 7 \\
5 \cdot 0 \\
5 \cdot 9 \\
7 \cdot 3\end{array}$ & $\begin{array}{l}39 \\
37 \\
27 \\
22 \\
15\end{array}$ & $\begin{array}{l}7 \cdot 6 \\
7 \cdot 2 \\
7 \cdot 3 \\
7 \cdot 2 \\
7 \cdot 7\end{array}$ & $\begin{array}{l}0.4 \\
0.4 \\
0.4 \\
0.5 \\
0.7\end{array}$ \\
\hline $\begin{array}{l}\text { Baseline } v \text { week } 0^{*} \\
\text { Week } 0 v \text { week } 12 \\
\text { Baseline } v \text { week } 12\end{array}$ & & & \multicolumn{2}{|c|}{$\begin{array}{l}<0 \cdot 001 \\
\text { NS } \\
\text { NS }\end{array}$} & \multicolumn{3}{|c|}{$\begin{array}{l}\text { ND } \\
\text { NS } \\
\text { ND }\end{array}$} & \multicolumn{3}{|c|}{$\begin{array}{l}<0 \cdot 001 \\
\text { NS } \\
\text { NS }\end{array}$} & \multicolumn{3}{|c|}{$\begin{array}{l}\text { NS } \\
\text { NS }\end{array}$} \\
\hline
\end{tabular}

*When repeated using only results from patients completing 12 weeks' treatment the significant results remained. When repeated usin only data from patients with a three day washout creatinine clearance still showed a significant change $(p<0 \cdot 01)$, but serum creatinine anథ serum urea did not.

${ }^{\dagger} \mathrm{NAG}=\beta-N$-acetylglucosaminidase. $N D=$ not determined (baseline, $\left.n=6\right)$; $N S=$ not significant $(p>0 \cdot 05)$ paircd $t$ test. 